\title{
Optimization of the collocation inversion method for the linear viscoelastic homogenization
}

\author{
Amna Rekik ${ }^{1}$, Renald Brenner ${ }^{2}$ \\ ${ }^{1}$ Institut PRISME (EA 4229, University of Orléans), Polytech'Orléans, France \\ ${ }^{2}$ LPMTM-CNRS, Univeristé Paris-Nord, 93430 Villetaneuse, France
}

\begin{abstract}
The effective behaviour of linear viscoelastic heterogeneous material can be derived from the correspondence principle and the inversion of the obtained symbolic homogenized behavior. Various numerical methods were proposed to carry out this inversion. The collocation method, widely used, within this framework rests on a discretization of the characteristic spectrum in a sum of discrete lines for which it is necessary to determine the intensities and the positions by the minimization of the difference between the exact temporal function and its approximation. The classical method is based on a priori choice of the lines positions and on the optimization of their intensities. It is shown here that the combined optimization of the positions and the (positive) intensities lead to a minimization problem under constraints. In the simple case of an incompressible isotropic twophase material, this method is confronted with the analytical solutions of the effective relaxation function and the classical collocation method. In the case of a continuous spectrum or a spectrum made up of a whole of discrete lines, the proposed method improves the predictions of the classical approach. For the various cases considered (ratio of the phases relaxation times, volume fraction of the components), a low number of the collocation points, even with the classical approach, lead to a good agreement with the analytical solution. The profit increases with the chosen interval time for the distribution of the collocation points especially for a spectrum made up of a whole of discrete lines.
\end{abstract}

Key words: Viscoelasticity; Homogenization; Laplace transform; Collocation method; Optimization

\section{Introduction}

To obtain the effective properties of non-ageing linear viscoelastic heterogeneous media, it is useful to apply the Laplace-Carson transform or the so-called correspondence principle [1] which helps to transform the linear viscoelastic behaviour into a symbolic elastic one for which it is possible to apply the classical linear homogenization techniques. The viscoelastic effective properties are then deduced by the inversion of the Laplace-Carson transform. Apart some simple cases, for which it is possible to carry out "exactly" this inversion by calculating the integral of Bromwich defined in the complex plane by $f(t)=\frac{1}{2 i \pi} \int \frac{f^{*}(p)}{p} e^{p t} d p$ [2], this inversion is usually performed numerically in an approached way. The majority of the work carried out within this framework rests on a development in series of the required temporal functions. We can in particular quote the widely used Prony-Dirichlet series or the collocation method developed by Schapery [8] and its extensions such as the multidata method proposed by Cost and Becker [5]. From a practical point of view, 
these methods rest on a preliminary choice of relaxation times (delays) and require the resolution of a linear system to determine the coefficients of the series approaching the relaxation (creep) function. Nevertheless, they do not impose conditions on these coefficients whereas a physical description of the stiffness (compliance) viscoelastic modulus requires positive coefficients. Various methods were proposed to overcome this difficulty [6,4], but they are always based on a preliminary adequate choice of the relaxation (creep) times.

This paper deals with the evaluation of the collocation method, used in homogenization [7], by considering a more general approach to approximate effective relaxation or creep function. To this end, we recall firstly the approximation of viscoelastic stiffness and compliance modulus by the Dirichlet series and we present the implemented procedure allowing to fit as well as possible the reference function. To assess the efficiency of this method, we consider then the case of an incompressible isotropic two-phase material for which analytical expressions of the effective behavior are available. This comparison enables especially to discuss the cases of relaxation functions with continuum spectra or made up of discrete lines and their consequence on the improved procedure.

\section{Principle of the improved collocation method}

According to the principle of superposition of Boltzmann, the local behavior in heterogeneous materials composed of linear viscoelastic constituents without ageing is governed by the following law:

$$
\sigma(t)=\int_{0}^{t} C(t-\tau) d \varepsilon(\tau) \quad \text { or } \quad \varepsilon(t)=\int_{0}^{t} S(t-\tau) d \sigma(\tau)
$$

where $C(t)$ is the tensor of viscoelastic modulus (i.e. relaxation function) and $S(t)$ is the tensor of viscoelastic compliances (i.e. the creep function). Based on the thermodynamics of the irreversible processes, Biot [3] showed that in the general case the linear viscoelastic compliance tensor reads:

$$
S(t)=S^{e}+S^{v} t+\int_{0}^{+\infty} J(\tau)\left(1-e^{-t / \tau}\right) d \tau
$$

where $J(t)$ is the spectrum of creep (or delay) of the material, $S^{e}$ and $S^{v}$ are the tensors of the elastic and viscous compliances characterizing the material's behavior, respectively, at $t=0$ and $t=+\infty$. The tensor of viscoelastic modulus is written:

$$
C(t)=\int_{0}^{+\infty} G(\tau) e^{-t / \tau} d \tau
$$

where $G(t)$ is the spectrum of relaxation of the material. Each characteristic spectrum, a priori unknown, is approached by a sum of discrete lines positioned at $n$ relaxation times or relaxation delays $\tau_{r}$. The relaxation function (3), as well as the transient part of the creep function $S=$ $S(t)-S^{e}-S^{v} t$ are thus approached by their expansion as Prony series as follows:

$$
C^{*}(t)=\sum_{r=1}^{n} G_{r} e^{-t / \tau_{r}}, \quad S^{*}(t)=\sum_{r=1}^{n} J_{r}\left(1-e^{-t / \tau_{r}}\right)
$$

where $G_{r}$ and $J_{r}$ are the intensities of the functions $C^{*}(t)$ and $S^{*}(t)$, respectively.

The determination of the coefficients and relaxation times of the transient series relies on the 
minimization of the quadratic error $E$ evaluated between the exact and approached functions. For the relaxation function, $E$ reads:

$$
E=\int_{0}^{+\infty}\left[C(t)-C^{*}(t)\right]^{2} d t
$$

whose minimization $\left(\partial E / \partial G_{r}=0\right.$ and $\left.\partial E / \partial \tau_{r}=0\right)$ leads to:

$$
\hat{C}\left(p_{r}\right)=\hat{C}^{*}\left(p_{r}\right) \quad \text { and }\left.\quad \frac{d \hat{C}(p)}{d p}\right|_{p_{r}}=\left.\frac{d \hat{C}^{*}}{d p}\right|_{p_{r}} \quad \text { where } \quad p_{r}=1 / \tau_{r}, \forall r=1, \ldots, n
$$

$\hat{\imath}$ denotes the Laplace transform. Substituting $C^{*}$ by its expression (4)-(a) leads to the following system:

$$
\hat{C}^{*}\left(p_{r}\right)=\sum_{i=1}^{n} G_{i} \frac{1}{p_{i}+p_{r}} \quad \text { and }\left.\quad \frac{d \hat{C}^{*}(p)}{d p}\right|_{p_{r}}=-\sum_{i=1}^{n} G_{i} \frac{1}{\left(p_{i}+p_{r}\right)^{2}} \quad \forall r=1, \ldots, n
$$

For a fixed number of terms of the the Dirichlet series, the best approximation of the exact function is thus obtained when the transforms of Laplace and their derivative are equal at $n$ points $p_{r}$. From a practical point of view, it is thus necessary to know the Laplace transform, as well as the derivative of this transform of the original temporal function at the various points of collocation. It is worth noting that the classical collocation method, resting on a preliminary choice of the points $p_{r}$, corresponds to the first equality (7)-(a) which defines a linear system allowing to determine the coefficients. For the improved collocation method, the obtained two equalities define a nonlinear system of $2 n$ equations (for each component of the viscoelastic modulus tensor) with $2 n$ unknown variables. In addition, it is necessary to introduce the following constraints for its resolution:

- The coefficents $G_{r}$ must be positive,

- The collocation times $\tau_{r}$ must be in the interval $\left[\tau_{\min }, \tau_{\max }\right]$,

- The relaxation function must be equal to the elastic modulus at the instant $t=0$.

The nonlinear system can then be written as a minimization problem under constraints:

$$
\left\{\begin{array}{l}
\min _{G_{r}, \tau_{r}}\left[\sum_{r=1}^{n}\left(\hat{C}\left(p_{r}\right)-\hat{C}^{*}\left(p_{r}\right)\right)^{2}+\sum_{r=1}^{n}\left(\left.\frac{d \hat{C}(p)}{d p}\right|_{p_{r}}-\left.\frac{d \hat{C}^{*}(p)}{d p}\right|_{p_{r}}\right)^{2}\right] \\
\text { where } \quad G_{r} \geq 0, \quad \tau_{\text {min }} \leq \tau_{r} \leq \tau_{\text {max }} \quad \text { and } \quad \sum_{r=1}^{n} G_{r}-C^{e}=0
\end{array}\right.
$$

where $C^{e}$ is the elastic modulus of the considered material. In a same manner, it is possible to define a minimization problem associated to the transient part of the creep function $S$. The former takes similar form than (8) but without the constraint on the behavior at $t=0$. In this work we will focus only on the relaxation function. To solve the minimization problem (8), we implement the algorithm Shor (SolvOpt) [10] which allows the optimization of non-smooth functions (case of a problem under constraints, for example).

\section{Illustrative example: case of a two-phase composite}

In order to evaluate the relevance of the numerical procedure of inversion proposed above and for the sake of simplicity, we consider in the following an isotropic material whose components obey 
an isotropic incompressible Maxwellian behaviour. The local behaviour of the phase $(i)$ is then described by the following compliance and stiffness tensors:

$$
S_{i}(t)=\left(m_{i}^{e}+m_{i}^{v} t\right) K \quad \text { and } \quad C(t)=2 \mu_{i}^{e} e^{-t / \tau_{i}} K+\infty J
$$

where $\tau_{i}=m_{i}^{e} / m_{i}^{v}$ and $2 \mu_{i}^{e}=1 / m_{i}^{e} . J$ and $K$ are the spherical and deviatoric isotropic projectors, respectively. The scalar constants $m_{i}^{e}$ and $m_{i}^{v}$ are, respectively, the elastic and viscous shear compliances of phase $(i), \mu_{i}^{e}$ is the elastic shear modulus and $\tau_{i}=m_{i}^{e} / m_{i}^{v}$ are the relaxation times characterizing the spectrum of the phase (i). The effective behavior of this composite is thus entirely defined by the scalar function $\tilde{\mu}(t)$.

Then applying the Laplace-Carson transform, the local behavior (1) becomes a symbolic elastic law characterized by the symbolic local shear modulus $\hat{\mu}_{i}^{*}(p)=p /\left[2 m_{i}^{e}\left(p+1 / \tau_{i}\right)\right]$. The effective symbolic shear modulus can therefore be evaluated using one of the available linear homogenization schemes such as the Mori-Tanaka or the self-consistent models. The expression of the shear modulus $\tilde{\mu}(t)$ is then obtained by the inversion of the Laplace-Carson transform of the symbolic result.

According to the Mori-Tanaka model, the analytical expression of the effective shear modulus reads [9]:

$$
\tilde{\mu}^{M T}(t)=\frac{b^{e}}{2 a^{v} m_{1}^{v}\left(\tau_{1}-\tau^{\prime}\right)}\left[\left(\frac{1}{\tau}-\frac{1}{\tau_{1}}\right) e^{-t / \tau_{1}}-\left(\frac{1}{\tau}-\frac{1}{\tau^{\prime}}\right) e^{-t / \tau^{\prime}}\right]
$$

where 2 denotes the inclusion phase, $\tau^{\prime}=\tau_{1}\left(2 c_{1}+\left(5-2 c_{1}\right) \frac{\tau_{2}}{m_{1}^{e} / m_{2}^{v}}\right)\left(2 c_{1}+\left(5-2 c_{1}\right) \frac{\tau_{1}}{m_{1}^{e} / m_{2}^{v}}\right), a_{v}=$ $5 m_{2}^{v}-2 c_{1}\left(m_{2}^{v}-m_{1}^{v}\right), b^{e}=5 m_{1}^{e}+3 c_{1}\left(m_{2}^{e}-m_{1}^{e}\right)$ and $c_{i}$ the volume fraction of the phase (i). According to this model, the spectrum of the relaxation function is thus composed of two discrete lines.

For the self-consistent model, Beurthey and Zaoui [2] showed that the spectrum of relaxation of the effective shear modulus consists of two discrete lines and a bounded continuous part of the form

$$
G^{A C}(\tau) \sim \frac{\tau_{1} \tau_{2}}{\sqrt{\theta_{1} \theta_{2}}} \frac{\sqrt{\left(\tau-\theta_{1}\right)\left(\theta_{2}-\tau\right)}}{\tau\left(\tau-\tau_{1}\right)\left(\tau_{2}-\tau\right)}
$$

as shown for example in figure 2-(a) (see [2]) where $\theta_{1}$ and $\theta_{2}$ are the bounds of the interval.

More details about the explicit expression of the shear modulus are given in [2]. In this work the integral expression of the shear modulus (11) was evaluated numerically. The result derived from the inversion method using the collocation method can then be confronted to the above quoted analytical results which differ mainly by the fact that the self-consistent model implies a continuous spectrum of relaxation.

\section{Results and discussion}

The "optimized" collocation method proposed in this paper is compared to the classical collocation method relying on a preliminary choice of the relaxation times and to the exact results provided by the self-consistent and Mori-Tanaka models for the relaxation function as shown on figures (1)(a)-(b) and figures (1)-(c)-(d), respectively. For figures (1)-(a) and (c), the relaxation times $\tau_{r}$ $(r=1, \ldots, n)$ characteristics of the collocation method are distributed in an interval qualified to be "minimal" $\left[\tau_{\min }, \tau_{\max }\right]=\left[\min \left(\tau_{i}\right), \max \left(\tau_{i}\right)\right]$ where $\tau_{i}$ are the relaxation times characteristics of the phase $(i)$. In figures (b) and (d) the relaxation times of the collocation method belong to an interval wider than the "minimal" interval. 
For relaxation times in the "minimal" interval, the results show that for a low number of points $(n \geq 3)$ the classical collocation method and its "optimized" version provide results in well agreement with the analytical solution characterized either by a continuous spectrum (figure (1)-(a)) or a discrete spectrum (figure (1)-(c)). It is noted that for a low number of relaxation times the "optimized" collocation gives the exact solution for relaxation function characterized by a spectrum made up of discrete lines as shown for the Mori-Tanaka scheme (see Figures (1-(c) and (d)). For relaxation times distributed in a wide interval, the "optimized" version of the collocation method considering a low number of points $(n \geq 8)$ for relaxation functions with continuous spectrum improves the results of its classical version.

The interest of the "optimized" collocation method increases if the relaxation function is characterized by a spectrum made up of discrete lines as it provides the exact solution. For a wide interval and with a high number of points $(n \geq 20)$ the classical collocation method provides estimates in good agreement with the exact solution. In opposition, for a low number of points, this method can provide negative estimates as shown on figure (1)-(d).

[Figure 1 about here.]

\section{Conclusion}

The combined optimization of the positions and the (positive) intensities of the Prony's serie approaching the exact relaxation (or creep) function improves the results of the classical collocation method with a low number of collocation points. The efficiency of the proposed method increases especially for the case of relaxation (creep) functions with a a spectrum made up of discrete lines. Indeed it provides exact solution for a low number of relaxation times. Such idea could be interesting for other homogenization models such as the generalized-self-consistent scheme or the " $N+1$ "-phase model (see for example [2]). Moreover, it can be applied for composites with compressible phases and extended for other existent numerical methods allowing the inversion of the Laplace-Carson transform.

\section{References}

[1] Mandel J., 1966, Mécanique des milieux continus. Gauthier-Villars, Paris.

[2] Beurthey, S., Zaoui, A., 2000. Structural morphology and relaxation spectra of viscoelastic heterogeneous materials. Eur. J. Mech. A/Solids 19, 1-16

[3] Biot, M. A., 1954. Theory of stress-strain relations in anisotropic viscoelasticity and relaxation phenomena. J. Appl. Phys. 25 (11), 1385-1391

[4] Bradshaw, R. D., Brinson, L. C., 1997. A sign control method for fitting and interconverting material functions for linearly viscoelastic solids. Mech. Time Dep. Matls. 1, 85-108

[5] Cost, T. L., Becker, E. B., 1970. A multidata method of approximate laplace transforminversion. Int. J. Num. Meth. Engng 2, 207-219

[6] Emri, I., Tschoegl, N. W., 1995. Determination of mechanical spectra from experimental responses. Int. J. Solids Struct. 32, 817-826 
[7] Laws, N., McLaughlin, R., 1978. Self-consistent estimates for the viscoelastic creep compliances of composite materials. Proc. R. Soc. Lond. A359, 251-273

[8] Schapery, R. A., 1962. Approximate methods of transform inversion for viscoelastic stress analysis. In : Proc. U.S. Nat. Congr. Appl. Mech. ASME 4th. Vol. 2. pp. 1075-1085

[9] Rougier Y., 1994. Étude du comportement sous irradiation: modélisation micromécanique de l'élastoviscoplasticité, PhD dissertation. École Polytechnique, Palaiseau, France.

[10] Website: http://www.kfunigraz.ac.at/imawww/kuntsevich/solvopt/ps/manual.pdf 


\section{List of Figures}

1 Relaxation functions evaluated by the improved and classical collocation methods compared to the exact results for a two-phase material according to the self-consistent scheme $(a-b)$ in the case $c_{2}=0.5, m_{1}^{e}=m_{2}^{e}=1, \tau_{2} / \tau_{1}=100$ and to the Mori-Tanaka scheme (c-d) in the case $c_{2}=0.5, m_{1}^{e}=m_{2}^{e}=1, \tau_{2} / \tau_{1}=10 \ldots \ldots \ldots$ 

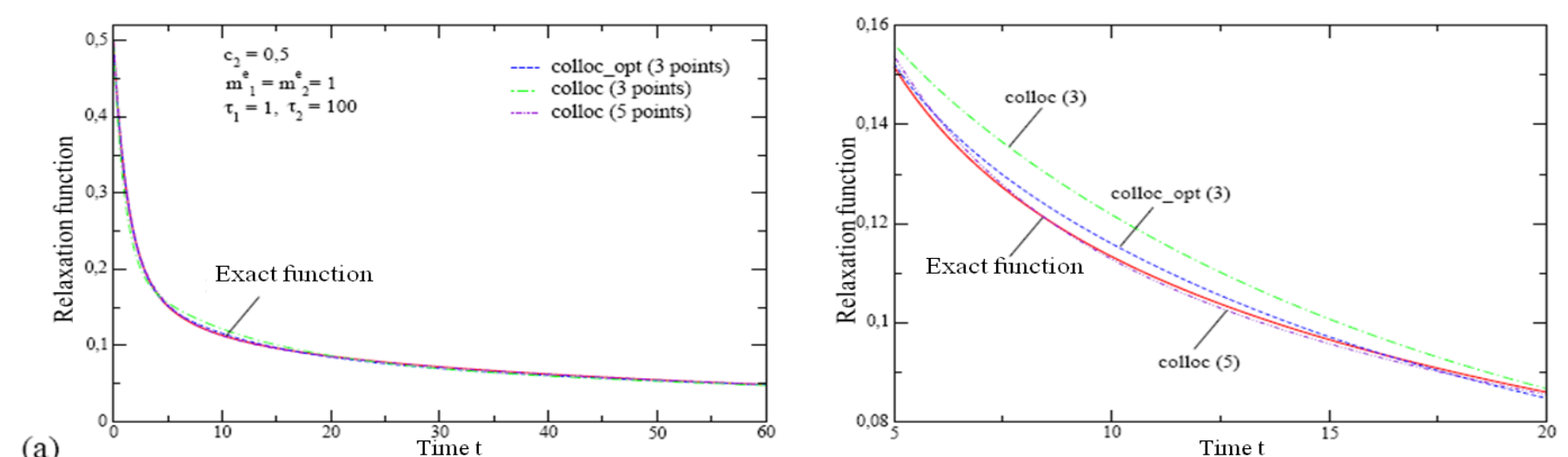

(a)

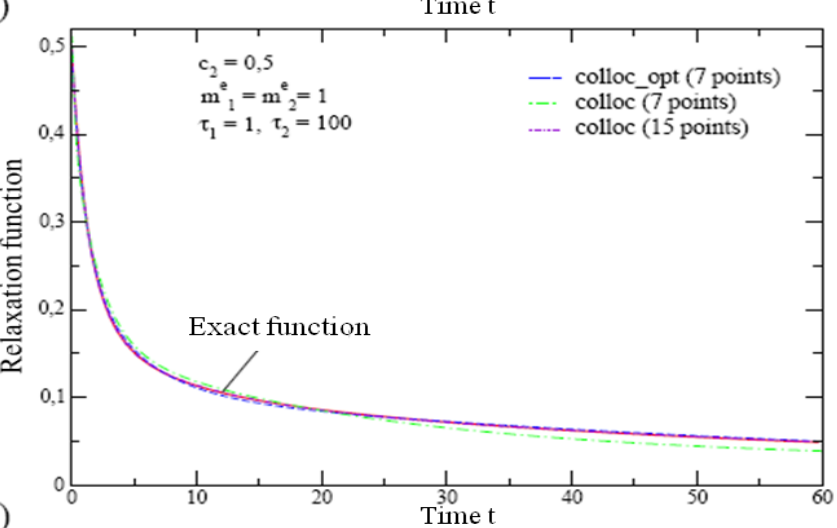

(b)

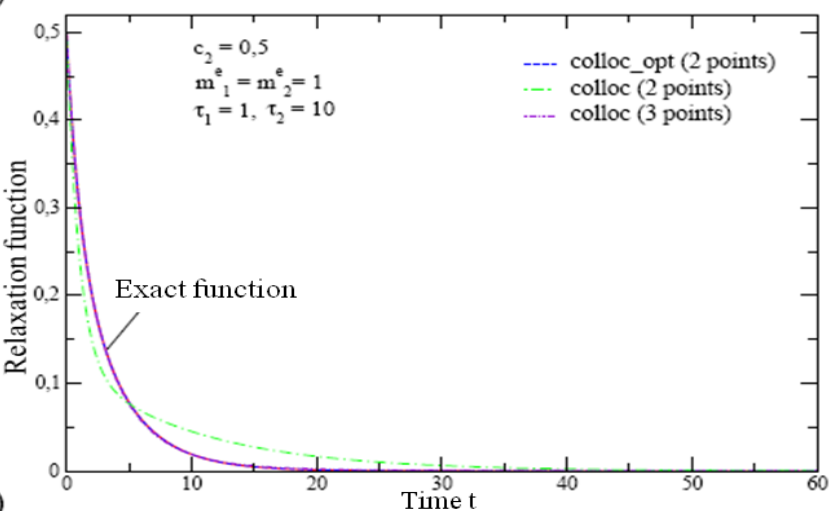

(c)

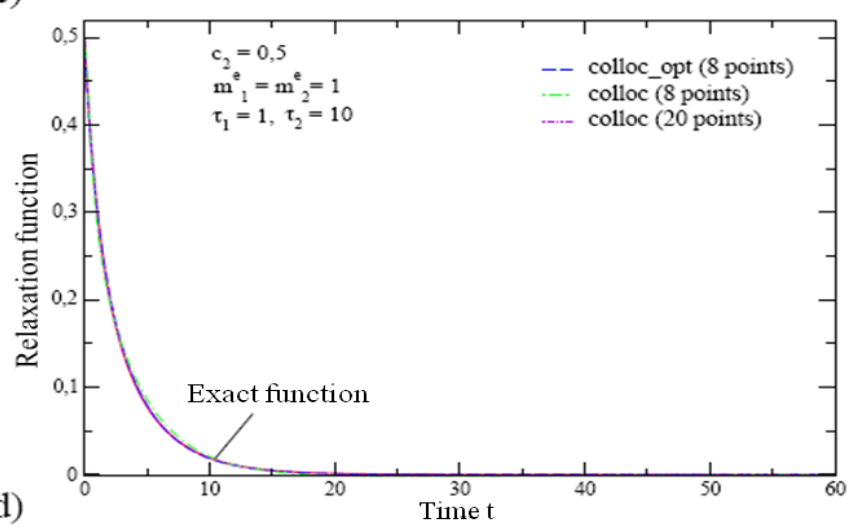

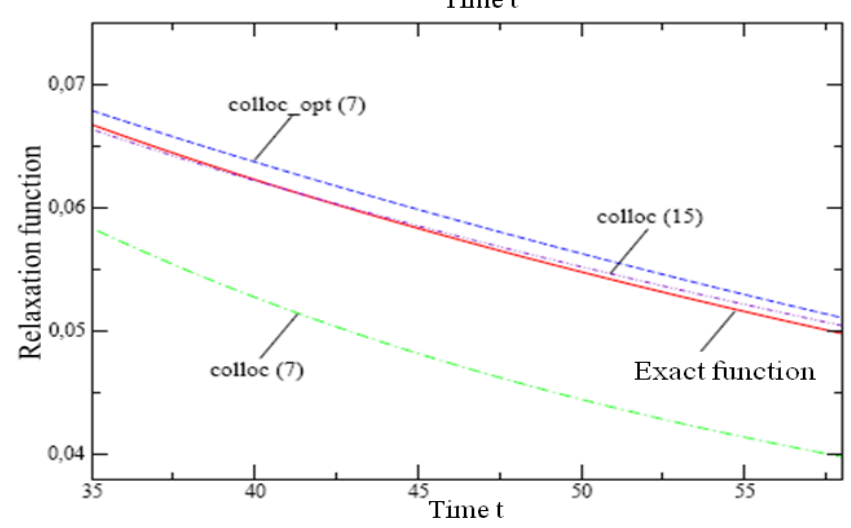
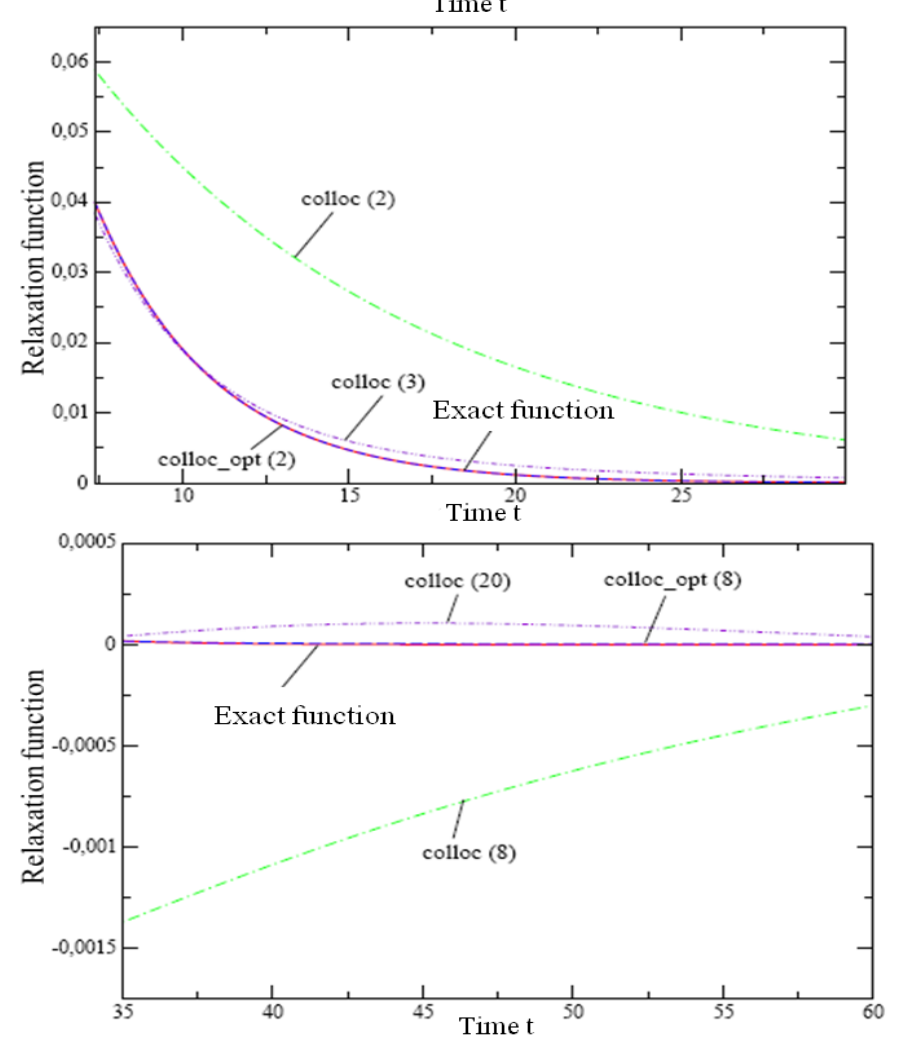

Figure 1: Relaxation functions evaluated by the improved and classical collocation methods compared to the exact results for a two-phase material according to the self-consistent scheme (a-b) in the case $c_{2}=0.5, m_{1}^{e}=m_{2}^{e}=$ $1, \tau_{2} / \tau_{1}=100$ and to the Mori-Tanaka scheme (c-d) in the case $c_{2}=0.5, m_{1}^{e}=m_{2}^{e}=1, \tau_{2} / \tau_{1}=10$. 